\title{
Mechanistic Analysis of Nucleophilic Substrates Oxidation by Functional Models of Vanadium-Dependent Haloperoxidases: A Density Functional Theory Study
}

\author{
Curtis J. Schneider, ${ }^{[a]}$ Giuseppe Zampella, ${ }^{[b]}$ Claudio Greco, ${ }^{[b]}$ Vincent L. Pecoraro, ${ }^{*[a, c]}$ and \\ Luca De Gioia*[b]
}

Keywords: Peroxido ligands / Tripodal ligands / Oxidation / Vanadium / Density functional calculations

Density functional theory has been used to investigate the structural, electronic, and reactivity properties of an established functional model for vanadium-dependent haloperoxidases, $\mathrm{K}\left[\mathrm{VO}\left(\mathrm{O}_{2}\right)\right.$ Hheida] (Hheida $^{2-}=2,2^{\prime}$-[(2-hydroxyethyl)imino]diacetate). Possible solution species were determined on the basis of potential exogenous donors present under the conditions necessary for reactivity. The energetically favored solution-state species is a 1:1 complex of Hheida and vanadium with a coordinated hydroxyethyl donor trans to the vanadium-oxido bond which is in agreement with the reported solid-state structure for $\mathrm{K}\left[\mathrm{VO}\left(\mathrm{O}_{2}\right)\right.$ Hheida]. Transition states of the oxidation reaction were located for four substrates: chloride, bromide, iodide, and dimethyl sulfide. The role of protonation and its effects on reactivity were examined for each substrate. Protonation of the peroxido moiety leads to a significant drop in the activation barrier for oxidation. In contrast no transition states could be located for an oxido-transfer process involving the oxido ligand. Barriers of activation calculated for halide oxidation were similar, providing support to the hypothesis that the $\mathrm{p} K_{\mathrm{a}}$ of the halide in acetonitrile is responsible for the decrease in reactivity between $\mathrm{I}^{-}, \mathrm{Br}^{-}$, and $\mathrm{Cl}^{-}$. The results presented herein provide a mechanistic correlation between a functional model and the enzyme, making $\mathrm{K}\left[\mathrm{VO}\left(\mathrm{O}_{2}\right)\right.$ Hheida] a "complete" functional model for vanadium-dependent haloperoxidase.

(๔ Wiley-VCH Verlag GmbH \& Co. KGaA, 69451 Weinheim, Germany, 2007)

\section{Introduction}

Vanadium complexes have been used in a number of oxidative transformations ${ }^{[1]}$ relevant to organic synthesis, including but not limited to alcohol oxidation, halide oxidation, and sulfoxidation. Of these reactions, the oxidation of achiral sulfides to chiral sulfoxides ${ }^{[1-4]}$ has sparked the most attention in recent years because of the potential applications of chiral sulfoxides. ${ }^{[5]}$ Peroxido-vanadium complexes are capable of the single oxidation of organic sulfides to sulfoxides with little double oxidation product (sulfone). ${ }^{[1,6,7]} \mathrm{A}$ number of catalysts have been presented in the literature ${ }^{[3,8-11]}$ to be competent with respect to sulfide oxidation and in some cases to be stereoselective. To date the best peroxido vanadium-based catalyst is $\mathrm{K}\left[\mathrm{VO}\left(\mathrm{O}_{2}\right)\right.$ Hheida], showing the highest rates of sulfide oxidation of any reported catalyst for bromide and organic sulfide oxidation. The mechanism of both halide and sulfide oxidation with this complex have been extensively studied. Thus, the $\mathrm{K}\left[\mathrm{VO}\left(\mathrm{O}_{2}\right)\right.$ Hheida] serves as an effective functional mimic

[a] Department of Chemistry, University of Michigan, Ann Arbor, Michigan, 48109-1055, USA

[b] Department of Biotechnology and Biosciences, University of Milano-Bicocca,

Piazza della Scienza 2, 20126 Milano, Italy

[c] Biophysics Research Division, University of Michigan, Ann Arbor, Michigan, 48109-1055, USA for the vanadium-dependent haloperoxidase class of enzyme and is an excellent catalyst in its own right.

Vanadium-dependent haloperoxidases (VHPO) are metalloenzymes containing a mononuclear vanadium $(\mathrm{V})$ cofactor $^{[12]}$ that catalyze the oxidation of halides in the presence of hydrogen peroxide. The oxidized species (HOX, $\mathrm{X}_{3}{ }^{-}$, or $\mathrm{X}_{2}$ ) subsequently adds to organic molecules, yielding halogenated marine products. ${ }^{[13]}$ The first example of this vanadoenzyme class was a bromoperoxidase isolated from the marine algae Ascophyllum nodosum. ${ }^{[14]}$ The enzyme nomenclature is based upon the most electronegative halide that the enzyme is capable of oxidizing. VHPO's can also catalyze the stereospecific two-electron oxidation of organic sulfides to chiral sulfoxides. ${ }^{[15]}$ The importance of a protonation of the active site for reactivity is suggested by the catalytically relevant $\mathrm{p} K_{\mathrm{a}}$ of $5.9^{[16]}$ for a vanadium bromoperoxidase. A number of X-ray crystal structures are now available for VHPO, ${ }^{[17-20]}$ including peroxide-bound and unbound forms of a vanadium chloroperoxidase from the fungus Curvularia inaequalis. ${ }^{[19]}$ The vanadate co-factor is covalently coordinated to an enzyme-bound histidine. The coordination geometry is trigonal bipyramidal, with an oxygen atom in the axial position trans to $\mathrm{N}$ atom of histidine, three other oxygen atoms complete the coordination sphere. The peroxide-bound form is best described as having a distorted tetragonal geometry when treating the per- 
oxido ligand as a single donor ligand. The coordination sphere is completed by an imidazole nitrogen donor, one oxido, and one hydroxido oxygen atoms.

Recently, computational methods have been employed to examine the resting and catalytically active states for simple models of the enzyme ${ }^{[21-26]}$ active site. The limitations of $\mathrm{X}$-ray crystallography left some ambiguity as to the protonation state of the vanadate co-factor in the resting and peroxido-bound forms. Density functional theory was used to explore the energetic consequences of protonation of a quantum chemical (QC) model for the vanadate co-factor with fixed nearby donor residues. ${ }^{[25]}$ The results of this study indicate the resting state to contain a cis-dioxido vanadium(V) species with one axial hydroxido group, an equatorial hydroxido, with an imidazole completing the first coordination sphere. Recent QM/MM calculations have indicated another isoenergetic potential resting state configuration that is stabilized by long range electrostatic interactions, and which contains three oxido moiety plus an axial water. ${ }^{[22,23]}{ }^{51} \mathrm{~V}$ solid state NMR spectroscopy was used in conjunction with DFT methods to examine the resting state configuration of vanadium chloroperoxidase; these studies confirm the resting state structure proposed in the aforementioned DFT and QM/MM studies. ${ }^{[21]}$

A number of mechanisms have been proposed for these enzyme based on small molecule mimics, including $\mathrm{KVO}\left(\mathrm{O}_{2}\right)$ Hheida, which generally invoke oxido-transfer catalysis. It has been shown that protonation of the peroxido moiety is essential for reactivity. Thus, in the catalytically active form there exists on the vanadium a terminal oxido and a protonated and unprotonated peroxido oxygen atoms. The question that then arises is which of these oxygen atoms is transferred to the substrate. ${ }^{[27]}$ Colpas et al. proposed that the transferred oxygen originated from the hydroperoxido moiety; however, these authors could not identify whether the protonated or unprotonated oxygen was involved. Computational methods have been employed to differentiate these two possibilities in QC models of VHPO. ${ }^{[26]}$ These studies indicate that oxido-transfer proceeded through the unprotonated peroxido atom through an $\mathrm{S}_{\mathrm{N}}$ 2-like process and that protonation of the peroxide ligand significantly lowered the barrier of activation for both halide and sulfide oxidation. These computational studies are in full agreement with reported catalytic abilities of the enzyme system. ${ }^{[16]}$

As previously mentioned, considerable effort has been expended to clarify the enzymatic mechanism, relatively little attention has been focused on the understanding the mechanism of the original catalysts described by Colpas and co-workers. These complexes are not only important for further our understanding of VHPO's, but in their own right as useful catalysts in organic synthesis. These vanadium tripodal amine complexes are the most effective sulfoxidation catalysts reported in the literature ${ }^{[6,27]}$ Mechanistic studies have shown the maximal rate of substrate oxidation occurs when one equivalent of proton with respect to vanadium is present. A red shift in the peroxido-vanadium charge-transfer band indicates the direct protonation of the vanadium complex. $\mathrm{V}^{51} \mathrm{NMR}$ showed a single resonance with a chemical shift and a linewidth consistent with a single mono-peroxido oxidovanadium( $(\mathrm{V})$ species under the reaction conditions; however, this does not rule out rapidly interconverting isoenergetic species. These observations were incorporated into a mechanistic proposal that was developed on the basis of the previously proposed halide oxidation mechanism, involving a hydroperoxido vanadium species undergoing an oxido transfer reaction with organic sulfides $^{[6]}$ (Figure 1). Despite these extensive mechanistic studies solution speciation under the reported reaction conditions, the site of catalyst protonation, the role of the protonated peroxido moiety in oxidative catalysis and the geometry of the transition state, still require further scrutiny.

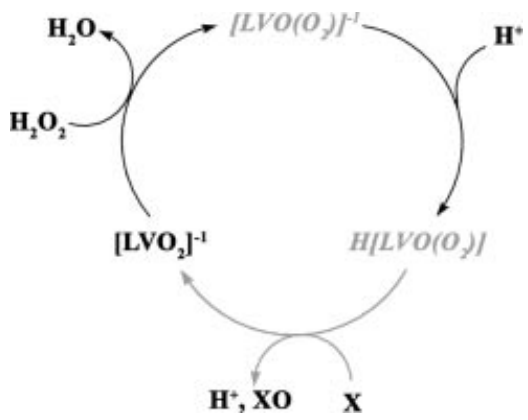

Figure 1. Diagram of the proposed mechanism for oxidation of a nucleophilic substrate by tripodal amino complexes of vanadi$\mathrm{um}(\mathrm{V}) . \mathrm{X}=\mathrm{Br}^{-}, \mathrm{I}^{-}$, or $\mathrm{R}_{2} \mathrm{~S}$. Steps highlighted in grey are the focus of this study.

Density functional theory has been used to address questions regarding reactivity and spectroscopic properties of transition-metal systems related to biology ${ }^{[28]}$ and catalysis. ${ }^{[29]}$ Balcells et al. reported computational studies of a truncated QC models for vanadium(V)-containing Schiff base complexes ${ }^{[30]}$ and its ability to oxidize organic disulfides in the presence of hydrogen peroxide. This study was later expanded to determine the factors controlling the stereoselectivity of disulfide oxidation using QM/MM methods ${ }^{[31]}$ on sterically hindered Schiff bases often employed in vanadium catalysis literature. ${ }^{[7,9-11,32]}$ Balcells et. al demonstrated the characteristics of an $\mathrm{S}_{\mathrm{N}}$ 2-like transition state, where oxido-transfer occurs from the unprotonated oxygen of the peroxido moiety to organic sulfides. Although the coordination environment of the Schiff base complex is significantly different from the enzyme system, it does bear some similarities (side-bound peroxido, similar transition state) and lends support to a peroxido atom transfer to the substrate. Schiff base vanadium(V) complexes have been shown to be functional mimics for VHPO based on their ability to oxidize bromide and organic sulfide, ${ }^{[9,11,32]}$ but kinetic analysis and, therefore, detailed mechanistic studies are not possible due to bromination of the ligand system. ${ }^{[33]}$

$\mathrm{K}\left(\mathrm{VO}\left(\mathrm{O}_{2}\right)\right.$ Hheida) has been studied extensively with respect to both bromide ${ }^{[27]}$ and sulfide ${ }^{[6]}$ oxidation, despite these synthetic results mechanistic questions relevant to future catalyst design based on these systems (i.e. protonation state of the catalytically active species and transition-state 
geometries). We present herein a detailed computational investigation of this catalyst. Treating the entire complex quantum mechanically, we provide a better understanding of the mechanism for substrate oxidation allowing more efficient catalyst design and providing a mechanistic correlation between these functional mimics and VHPO's.

\section{Results}

\section{Quantum Chemical System}

The X-ray coordinates for $\mathrm{K}\left[\mathrm{VO}\left(\mathrm{O}_{2}\right) \text { Hheida }\right]^{[34]}$ were used as a starting point to set up a quantum chemical model of this system. Waters of crystallization were removed, as was the potassium ion. Under the reaction conditions used by Colpas et al., there are two equivalents of 18-crown- 6 which chelate the potassium ion in acetonitrile, limiting the potential interactions of the cation with the vanadium complex during the catalytic cycle. The resulting

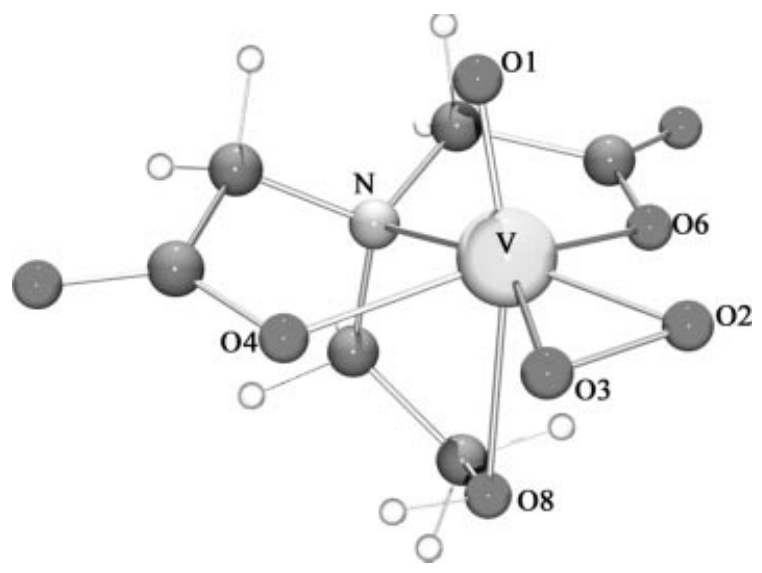

Figure 2. Ball-and-stick representation of geometry optimized $\mathbf{1}$.

Table 1. Bond lengths $[\AA ̊]$ of $\left[\mathrm{VO}\left(\mathrm{O}_{2}\right) \text { Hheida }\right]^{-1}$.

\begin{tabular}{lcc}
\hline & X-ray structure & Optimized $\mathbf{1}$ \\
\hline V-O1 & 1.60 & 1.62 \\
V-O2 & 1.87 & 1.85 \\
V-O3 & 1.86 & 1.85 \\
V-O4 & 2.05 & 2.10 \\
V-O6 & 2.04 & 2.05 \\
V-O8 & 2.24 & 2.45 \\
V-N1 & 2.19 & 2.28 \\
O3-O2 & 1.43 & 1.44 \\
\hline
\end{tabular}

energy-minimized geometry of $\left[\mathrm{VO}\left(\mathrm{O}_{2}\right) \text { Hheida }\right]^{-1}(\mathbf{1})$, depicted in Figure 2 is nearly identical to the bond lengths reported in the crystal structure (Table 1).

\section{Protonation and Solution Speciation}

Before exploring the oxidative chemistry of $\mathbf{1}$, a better understanding of the solution-state speciation for this precatalytic species is necessary. The crystal structure of $\mathrm{K}\left[\mathrm{VO}\left(\mathrm{O}_{2}\right)\right.$ Hheida] shows a disordered hydroxyethyl arm due to two ring conformation of a five-membered chelate ring. The interconversion between these two states is rapid compared to the rate of oxidation, therefore, the left- and right-hand sides of $\mathbf{1}$ are treated as equivalent for optimizations involving an $\mathrm{Hheida}^{-2}$ ligand. The weakest coordinating and therefore most labile donor atom is the oxygen associated with the hydroxyethyl arm of Hheida ${ }^{2-}$. Geometryoptimized structures were obtained initially both with a coordinated hydroxy oxygen atom and a completely dissociated hydroxy group. The former being the more stable of the two by $1.3 \mathrm{kcal} / \mathrm{mol}$. Given the two potential pre-catalytic isomers, a coordinated hydroxy and the uncoordinated hydroxy group and the presence of sigma donor ligands under the reaction conditions (water, acetonitrile, and substrate), a vacant coordination site is not likely. As there is no experimental evidence supporting coordination of substrate molecules in VHPO or in the small molecule models, substrate coordination was ruled out as possibility. A comparison of the energetic differences between the van der Waals complex of the exogenous donors, water and acetonitrile, with the coordinated exogenous donor in place of the hydroxy group was made. These comparisons were made using the most stable protonated species to reduce the electronic repulsion created by the approach of a sigma donor to an anionic vanadium species. The results of this comparison are presented later.

Five potential sites of protonation were identified on the basis of the areas of highest electron density for $\mathbf{1}$ : oxido $(\mathbf{0 c})$, peroxido left $(\mathbf{0 b})$, peroxido right $(\mathbf{0 a})$, carboxylate oxygen (0d), and the carbonyl oxygen (0e) (Figure 3). Only one of the carboxylate donors was protonated due to the previously mentioned conformational isomerism. Protonation of the peroxido can occur in four possible locations as illustrated in part b of Figure 3. The most stable optimized structures involved protonation of the peroxido ligand $\mathbf{0 a}$ or $\mathbf{0 b}$. The four possible orientations of the proton around
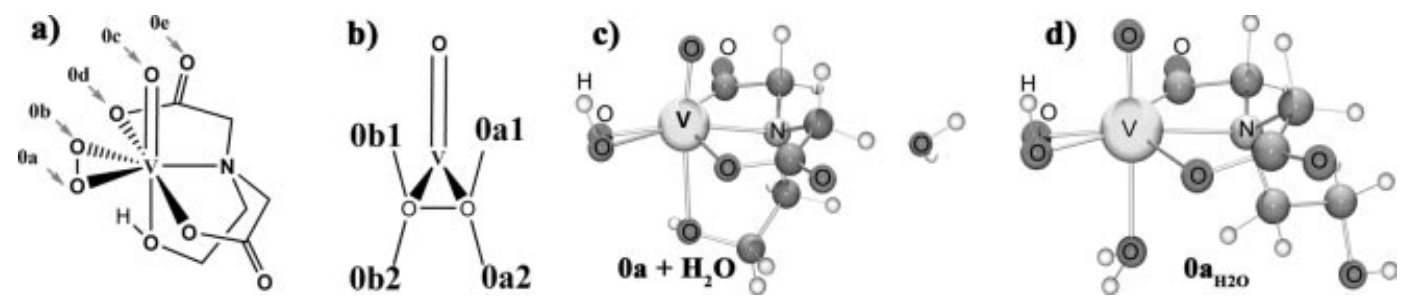

Figure 3. (a) Proton acceptors in 1. (b) Nomenclature for the orientation of proton with respect to V=O bond (c) Representative ball-andstick diagram of a non-coordinated exogenous donor, in this the exogenous donor is water. (d) Representative cartoon for coordination of an exogenous donor to vanadium. White atoms are hydrogens and unlabeled atoms are carbon. 
the peroxido ligand were isoenergetic when ring conformations were taken into account. There is significant contraction of the vanadium hydroxy bond (V-O8) upon protonation of the complex in $\mathbf{0 a}, \mathbf{0 b}$, or $\mathbf{0 c}$, a decrease of $0.1 \AA$ for $\mathbf{0 a}$ and $\mathbf{0 b}$, and $0.2 \AA$ for $\mathbf{0 c}$.

The most stable optimized geometry, $\mathbf{0 a}$, was used to determine whether exogenous ligands can bind trans to the oxido moiety. Three exogenous donors were tested in place of hydroxy coordination to the vanadium center: water, acetonitrile, and bromide. In all cases, a van der Waals complex of the exogenous donor and $\mathbf{0 a}$ is more stable than coordination of the respective donor in place of the hydroxy. In the case of water coordination to 0a, (Figure 3, parts c, d), hydroxy coordination to vanadium over water was favored by $4.0 \mathrm{kcal} / \mathrm{mol}$. A coordinatively unsaturated species, dissociation of the hydroxy group with no trans donor, was less favored by $4.6 \mathrm{kcal} / \mathrm{mol}$.

\section{Halide Transition States}

Transition states corresponding to the nucleophilic attack of halide ions $\left(\mathrm{Cl}^{-}, \mathrm{Br}^{-}, \mathrm{I}^{-}\right)$on the peroxido ligand of the vanadium atom were located for both $\mathbf{1}$ and 0a. Due to the anionic character of both the halide and $\mathbf{1}$, starting materials were calculated on the basis of two infinitely separated species, as any attempt to minimize a van der Waals complex resulted in the two ions rapidly diverging. The transition-state geometry found for $\mathbf{1}$ with all three halides (1_cl, 1_br, 1_i) are nearly identical, therefore, a representative case will be presented for the oxidation of bromide. The transition state for bromide oxidation is characterized by a linear halide peroxido bond angle $\left(173^{\circ}\right)$, where the dihedral angle between the peroxido ligand and the vanadium-oxido is $100^{\circ}$ (Figure 4). The rest of the vanadium coordination sphere is significantly perturbed in comparison to $\mathbf{1}$, there is a dramatic lengthening of all bonds in the first coordination sphere, in the case of $\mathbf{1}$ br the carboxylate donors have bond lengths of $2.27 \AA$ whereas the carboxylate-vanadium distances in $\mathbf{1}$ are $2.04 \AA$. Additionally the tertiary nitrogen bond lengthened by $0.3 \AA$ in relation to 1 (Table 2). The computed barrier of activation, $\Delta G^{\ddagger}$, for bromide oxidation is $38.1 \mathrm{kcal} / \mathrm{mol}$.
Table 2. Bond lengths $[\AA]$ of the transition states for bromide oxidation.

\begin{tabular}{lccc}
\hline & $\mathbf{1}$ & $\mathbf{1}$ _br & 0a_br \\
\hline V-O1 & 1.62 & 1.64 & 1.62 \\
V-O2 & 1.85 & 1.71 & 2.05 \\
V-O3 & 1.86 & 1.90 & 1.90 \\
V-O4 & 2.10 & 2.27 & 2.02 \\
V-O6 & 2.05 & 2.27 & 1.99 \\
V-O8 & 2.45 & 2.48 & 2.30 \\
V-N1 & 2.28 & 2.50 & 2.29 \\
O3-O2 & 1.44 & 1.87 & 1.69 \\
O3-Br & - & 2.27 & 2.38 \\
\hline
\end{tabular}

Protonation of $\mathbf{1}$ at the most stable site (0a) yields an earlier transition state (0a_br), in which the $\mathrm{Br}-\mathrm{O}$ bond is only partially formed with a nearly complete $\mathrm{O}-\mathrm{OH}$ bond in the peroxido moiety, with a significantly lower barrier of activation $(21.1 \mathrm{kcal} / \mathrm{mol})$. The bromide-peroxido oxygen bond lengths is longer by $0.11 \AA ̊$ than $\mathbf{1} \_\mathbf{b r}$, with a comparably shorter peroxide bond length. The $\mathrm{Br}-\mathrm{O} 3-\mathrm{O} 2$ bond angle is $163.4^{\circ}$ while the dihedral angle between the peroxido ligand and the vanadium-oxido is $123.8^{\circ}$. Transitions states were only located in the protonated case where a hydrogen bond formed between the halide and the hydroxy ligand. No transition states related to halide oxidation could be located for attack of the substrate on the protonated peroxido ligand. This is true for all anionic halide transition states (0a_cl, 0a_br, and $\mathbf{0 a} \mathbf{i})$, where the corresponding barriers to activation are $23.0 \mathrm{kcal} / \mathrm{mol}$ for $\mathrm{Cl}^{-}$and $19.0 \mathrm{kcal} /$ mol for $\mathrm{I}^{-}$.

To explore the reactivity of $\mathbf{0 a} \_\mathbf{x}$ further, a coordinatively unsaturated geometry was used as the starting point, in which a halide can weakly coordinate to vanadium(V) center. Bromide was used as the substrate and positioned to weakly coordinate to the vanadium center. $\mathbf{0 a} \_\mathbf{b r} \mathbf{2}$ is characterized by a distortion of the dihedral angle $\mathrm{V}-\mathrm{O} 1-\mathrm{O} 2-\mathrm{O} 3$ $\left(149^{\circ}\right)$ allowing a weak interaction of the bromide with vanadium $3.03 \AA$ A. A reasonable reactant structure could be located with bromide coordinating to the vanadium, and the computed barrier of activation is $8.6 \mathrm{kcal} / \mathrm{mol}$. The barrier is significantly lower in energy because the coordination of bromine to an coordinatively unsaturated vanadium complex (0a_br2) is $10 \mathrm{kcal} / \mathrm{mol}$ higher energy than the corresponding hydrogen-bonded starting materials (0a_br).
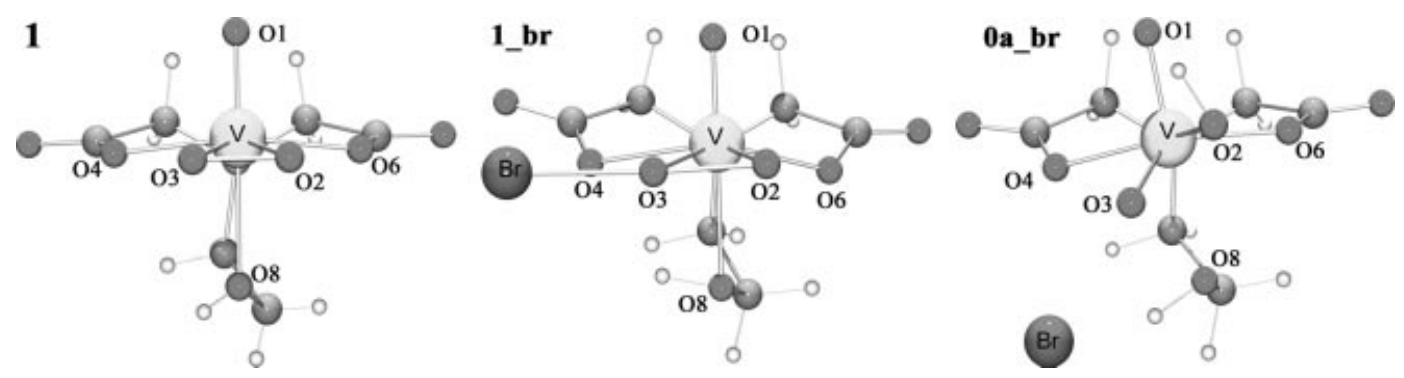

Figure 4. Ball-and-stick cartoons showing pre-catalytic anionic complex (left), dianionic transition state for bromide oxidation (middle) and the corresponding monoanionic transition state. 
Table 3. Comparison of relevant bond lengths and $\Delta E_{\mathrm{scf}}[\mathrm{kcal} / \mathrm{mol}]$ for transition states associated with halide oxidation.

\begin{tabular}{|c|c|c|c|c|c|c|c|c|c|}
\hline & 1 & 1a_cl & 1a_br & 1a_i & $\mathbf{0 a}$ & Oa_cl & 0a_br & Oa_i & 0a_br2 \\
\hline O3-X & - & $2.17 \AA$ & $2.27 \AA$ & $2.39 \AA$ & - & $2.29 \AA$ & $2.38 \AA$ & $2.55 \AA$ & $2.48 \AA$ \\
\hline $\mathrm{O} 3-\mathrm{O} 2$ & $1.44 \AA$ & $1.85 \AA$ & $1.87 \AA$ & $1.90 \AA$ & $1.46 \AA$ & $1.77 \AA$ & $1.69 \AA$ & $1.69 \AA$ & $1.70 \AA$ \\
\hline $\mathrm{V}-\mathrm{O} 1-\mathrm{O} 2-\mathrm{O} 3$ & $98.5^{\circ}$ & $100.3^{\circ}$ & $100.0^{\circ}$ & $100.0^{\circ}$ & $103.7^{\circ}$ & $124.5^{\circ}$ & $123.7^{\circ}$ & $121.8^{\circ}$ & $149.0^{\circ}$ \\
\hline$\Delta E_{\mathrm{scf}}^{\dagger}[\mathrm{kcal} / \mathrm{mol}]$ & - & 39.2 & 38.1 & 36.0 & - & 23.0 & 21.1 & 19.0 & 20.05 \\
\hline
\end{tabular}

The geometries and respective energy differences between starting materials and transition states for all located halide transition states are presented in Table 3. No transition states could be located corresponding to attack of the halide on the oxido ligand of $\mathbf{1}, \mathbf{0 a}$, or $\mathbf{0 c}$.

\section{Sulfide Transition States}

The effects of protonation on the barrier of activation were also explored for the oxidation of dimethyl sulfide. Transition states calculated for dimethyl sulfide oxidation are less distorted from the energy minimized structures of $\mathbf{1}$ or the appropriate protonated species $\mathbf{0}(\mathbf{a}, \mathbf{b})$ than the corresponding halide transition state. Figure 5 shows two representative transition states located for sulfide oxidation by anionic and neutral vanadium species.

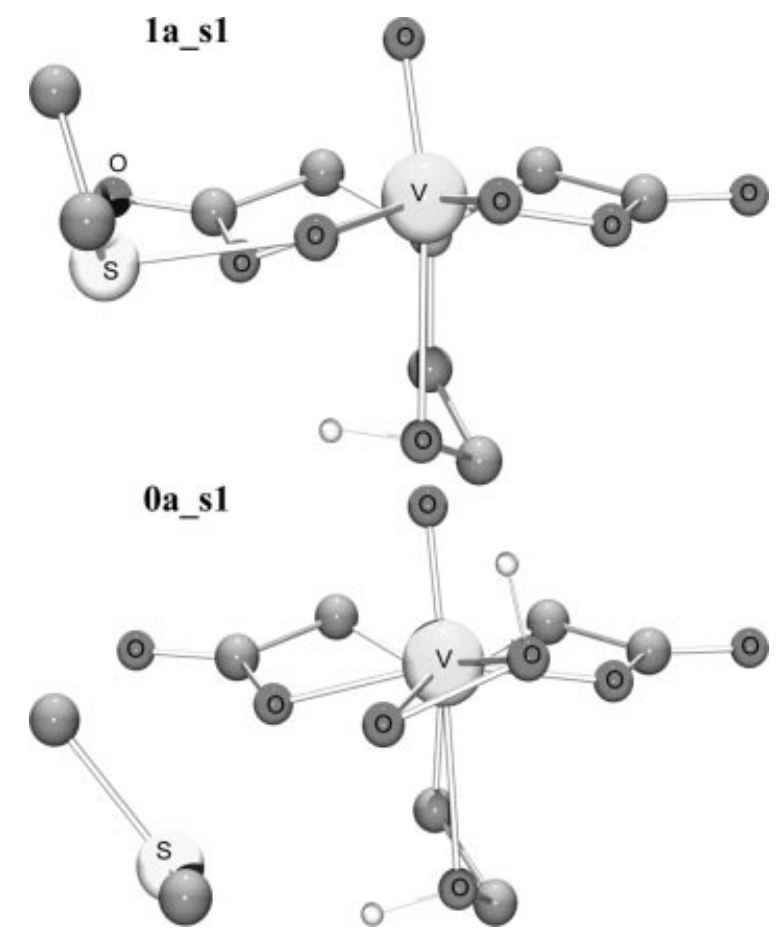

Figure 5. Ball-and-stick representation of an anionic (top) and neutral (bottom) transition state located for dimethyl sulfide oxidation.

The transition-state geometry 1_s is characterized by a nearly linear S-O2-O3 bonding angle, identical to that identified for the case of halide oxidation. Due to the asymmetry associated with upper and lower halves of $\mathbf{1}$, multiple transition states with slightly different energies could be located, the only difference being related to relative orientation of the methyl groups of dimethyl sulfide. Approach of dimethyl sulfide to the left or right hand side of $\mathbf{1}$ yielded nearly isoenergetic transition states, with a barrier of activation of $18.3 \mathrm{kcal} / \mathrm{mol}$. Starting materials were minimized from each located transition state generating a van der Waals complex of dimethyl sulfide and $\mathbf{1}$.

Transition states were located for the protonated complexes $\mathbf{0 a} \_\mathbf{s}$ and $\mathbf{0 b} \mathbf{b}$. As was the case for $\mathbf{1}$, multiple rotational isomers could be located and are related to orientation of the methyl groups on the substrate. The neutral transition states located for dimethyl sulfide oxidation are less distorted than $\mathbf{0 a} \_\mathbf{X}$, with dihedral angles approx. V$\mathrm{O} 1-\mathrm{O} 2-\mathrm{O} 3111.8^{\circ}$ and a S-O3-O2 of $166.6^{\circ}$ where the sulfide attacks the unprotonated peroxido oxygen. To calculate the barrier of activation, van der Waals complexes of dimethyl sulfide and the appropriately protonated vanadium species were optimized. The lowest energy starting material complex located contained a hydrogen bond forms between dimethyl sulfide and the hydroxy group of the complex the barrier of activation for the oxidation reaction is $9.4 \mathrm{kcal} /$ mol. The barrier of activation drops significantly (by $9 \mathrm{kcal} /$ $\mathrm{mol}$ ), when the peroxido moiety is protonated, leading to four isoenergetic transition coordinates differing in the approach of sulfide to the neutral vanadium complex and orientation of the sulfide. No transition states could be located related to attack of the sulfide on the unprotonated oxido group $(\mathbf{0 c})$ or attack of the sulfide on the protonated peroxido oxygen.

\section{Discussion}

Although many model complexes ${ }^{[27,32,33,35,36]}$ have been presented in the literature, the pre-catalytic species $\mathrm{K}\left[\mathrm{VO}\left(\mathrm{O}_{2}\right) \text { Hheida }\right]^{[34]}$ isolated by Colpas and co-workers is the most thoroughly investigated system. Detailed mechanistic studies have shown the importance of protonation of the complex with respect to halide ${ }^{[27]}$ and sulfide oxidation. ${ }^{[6]}$ These studies established $\mathrm{K}\left[\mathrm{VO}\left(\mathrm{O}_{2}\right)\right.$ Hheida] as a functional mimic of the vanadium-dependent haloperoxidases and as an excellent catalyst for organic transformations. We have undertaken a thorough computational investigation of the mechanism for halide and sulfide oxidation, specifically with respect to the effects of protonation of the complex on the activation barrier for these processes in order to develop future asymmetric oxidation catalysts based on the $\mathrm{H}_{3}$ heida architecture.

Starting coordinates are taken from the crystallographically characterized pre-catalyst, we optimized chemically reasonable solution state species. The crystal structure of $\mathrm{K}\left[\mathrm{VO}\left(\mathrm{O}_{2}\right)\right.$ Hheida] shows a hydroxy group trans to the ox- 
ido, with tertiary nitrogen trans to the peroxido moiety, a typical binding mode for oxidovanadium(V) peroxido complexes with tripodal amino ligands. The experimental conditions have been taken into account when setting up the QM investigation, both regarding solvent polarizing power, and to explore the possibility of binding by exogenous donor ligand to vanadium. Potassium is chelated by $18-\mathrm{C}-6$ allowing $\mathrm{K}\left[\mathrm{VO}\left(\mathrm{O}_{2}\right)\right.$ Hheida] to be solubilized in acetonitrile. On the basis of this condition, the quantum chemical model, 1, excluded potassium in all geometry optimizations. The weakest donor group in $\mathbf{1}$ is the hydroxy group trans to the oxido. As there is no ligand field stabilization energy for a vanadium $(\mathrm{V})$ complex, a coordinatively unsaturated species is potentially feasible. Geometry optimization was performed on an anionic complex in which the hydroxy group was completely dissociated from the complex, the resulting optimized structure is $1.3 \mathrm{kcal} / \mathrm{mol}$ higher in energy when enthalpic and entropic corrections are taken into account. Under the reaction conditions a number of exogenous donors are present in at least equimolar quantities with respect to the vanadium complex, making a coordinately unsaturated species less likely.

Before examining the energetic preference for exogenous donors, we explored the energetics of monoprotonated 1. The potential proton acceptors in the complex are all of the oxygen atoms associated with the ligand, this generates five potential sites of protonation. Protonation of the peroxido ligand is the most energetically favored, there is a slight difference between protonation of $\mathrm{O} 3$ and $\mathrm{O} 2$ this effect is negligible when chelate ring conformations of the hydroxyethyl arm of $\mathbf{1}$ are taken into account. Protonation of the peroxido ( $(\mathbf{0}$ and $\mathbf{0 b})$ is favored by $3 \mathrm{kcal} / \mathrm{mol}$ over protonation of the oxido ligand $(\mathbf{0 c})$, while protonation of either carboxylate oxygen generates a species $6 \mathrm{kcal} / \mathrm{mol}$ higher in energy.

Using the energetically favored protonation states of the complex, two optimizations were performed for exogenous donors, one in which a van der Waals complex of the donor was made with a coordinated hydroxy species $\mathbf{0 a}$, and one with the exogenous donors coordinated in place of the hydroxy. For all the exogenous donors tested (water, acetonitrile, and bromide), the van der Waals complex was more stable by 2,7 , and $15 \mathrm{kcal} / \mathrm{mol}$, respectively. Thus a coordinated hydroxy species is likely to be the dominate solution species present. Previously ${ }^{51}$ V NMR has been used to explore the number of vanadium species present under the reaction conditions, suggesting only one species is present or there is an exchange process faster than the ${ }^{51} \mathrm{~V}$ NMR timescale. All factors considered, the loss of entropy is higher in the cases of exogenous donor molecules. Since the enthalpic contribution is roughly similar when considering exogenous and endogenous ligands (on binding to $\mathrm{V}$ ) a larger gain in the entropy is expected when a water, bromide, or other exogenous ligand dissociates, compared to an endogenous ligand.

Using the most stable unprotonated (1) and protonated species (0a) as a starting point, transition-state searches were performed for chloride, bromide and iodide. Transi- tion state located for $\mathbf{1}$ showed a significant lengthening of all ligand bonds to the vanadium, due to the increased electron density of the complex in the transition state. All bond lengths are still within a weak bonding distance. The transition state corresponded to the $\mathrm{S}_{\mathrm{N}} 2$-like attack on the peroxido ligand of the vanadium complex as determined by examining the single negative vibrational eigenvalue. No transition state could be located for bromide attack on the vanadium-oxido bond. The energy of $\mathbf{1}$ and the appropriate halide at infinite separation were used as starting materials, as any attempt to generate a van der Waals complex resulted in a rapid divergence of the two anions. The resulting energies of activation were 39,38 , and $36 \mathrm{kcal} / \mathrm{mol}$ for chloride, bromide, and iodide, respectively.

Upon protonation of the complex, a pre-reaction adduct is formed, in which a hydrogen bond between the halide ion and the complex is present and, most importantly, the barriers of activation decreased dramatically. The resulting monoanionic transition states are $\mathrm{S}_{\mathrm{N}} 2$-like with the nearly linear $\mathrm{V}-\mathrm{O} 3-\mathrm{O} 2 \mathrm{H}$ bond angle and have strongly distorted $\mathrm{V}-\mathrm{O} 1-\mathrm{O} 3-\mathrm{O} 2$ dihedral angle. The hydrogen bond is maintained in all anionic halide transition states, and no transition states could be located for complexes in which this hydrogen bond was not present. Due to the large electron density surrounding the carboxylate donors of $\mathbf{0 a}$, it is necessary to stabilize the negative charge of the halide in this early transition state. Under the experimental reaction conditions this stabilization could be accomplished by the presence of hydrogen-bond donors (water or hydrogen peroxide) or cations (tetraethylammonium or potassium).

Zampella et al. located transition states for the oxidation of bromide using quantum chemical models of the active site of a VHPO. ${ }^{[26]}$ Their results showed that nucleophilic attack of the halide on the unprotonated oxygen of a hydroperoxido species had the lowest barrier of activation. Transition states were located for the peroxido vanadium adduct; protonation of the peroxido moiety yielded a significantly more stable transition geometry resulting in a lower barrier of activation. The hydroperoxido transition state occurred significantly earlier with respect to halide-oxygen formation than the peroxido transition state. The transition states located for bromide oxidation by $\mathbf{1}$ and $\mathbf{0 a}$ show the same trend with respect to the halide-oxygen bond formation. The resulting transition state $\mathbf{0 a}$ abr shows a longer halide-oxygen bond than 1_br, indicating an earlier transition state. Additionally, there is dramatic decrease in the barrier of activation from 1_br to $\mathbf{0 a}$ a_br (Table 3); this observed decrease in the barrier of activation is likely an oversimplification of experimental barrier because of the interaction between two anionic species (vanadium complex and the halide) in the case of 1_br. Indeed, under reaction conditions it may be possible to carry out this reaction by offsetting the negative charge with strongly associated cations or hydrogen-bond donors. These results now correlate the mechanism of halide oxidation for the best reported functional model for VHPO, $\mathrm{K}\left[\mathrm{VO}\left(\mathrm{O}_{2}\right)\right.$ Hheida], with the proposed mechanism for halide oxidation by the enzyme. The importance of offsetting the negative charge on the anionic 
halide is observed in the case of $\mathbf{0 a} \_\mathbf{b r}$ transition state, where a hydrogen bond forms between the bromide and the hydroxy group of the ligand resulting in a distorted dihedral angle for the peroxido moiety with respect to the vanadium-oxido bond. This is further supported by the location of a nearly isoenergetic reaction pathway, in which the bromide weakly coordinates to coordinatively unsaturated vanadium species 0a_br2.

Given that VClPO is capable of oxidizing chloride, bromide and iodide, and $\mathrm{K}\left[\mathrm{VO}\left(\mathrm{O}_{2}\right)\right.$ Hheida] has been shown to be competent with respect to only bromide and iodide oxidation, we chose to explore the differences in barrier of activation for the oxidation of the aforementioned halides. Reactivity studies demonstrate the protonated $\mathrm{K}\left[\mathrm{VO}\left(\mathrm{O}_{2}\right)\right.$ Hheida] is capable of oxidizing bromide and iodide, but not chloride. Two possible factors could contribute to this inability to oxidize chloride: 1 . The reduction potential of the chloride is sufficiently high to prevent oxidation, 2. The active protonated form of the complex can not be generated in presence of chloride. The latter hypothesis was presented by Colpas et al. to explain this reactivity. ${ }^{[27]}$ Slebodnick et al. demonstrated the ability of a tripodal amino complex to oxidize chloride when a mixture of acetonitrile $(94 \%)$ and water $(6 \%)$ was used as the solvent system. ${ }^{[37]}$ Because chloride oxidation is not observed in either pure solvent it appears that in a mixture of the two solvents the basicity of the peroxido vanadium species increases with respect to chloride, allowing the catalytically active protonated species to be formed. On the basis of the transition-state energies presented herein, there is not a large difference in the energies of activation for each of the halides, therefore, differences in the reactivity can not be explained solely on the differences in reduction potentials of the oxidized halides. As protonation of the complex generates an early transition state, in which formation of the halide-oxygen bond is very weak, there is less contribution of halide orbital character in the transition state leading to smaller differences in the barrier of activation for halide oxidation (Table 3). These energies of activation presented here provide further support for this hypothesis.

To complete the functional correlation between the quantum-chemical models of the enzyme system and functional models for the enzyme, we examined the effects of protonation of the complex on organic sulfide oxidation. In the case of halide oxidation, the effects of protonation on the reaction barriers are masked to some degree by a highly charged dianionic transition state in the case of $\mathbf{1} \_\mathbf{x}$, although this does represent the experimental conditions quite well, considering the chelation of potassium ions by 18-crown-6 preventing strong ion pair formation.

The organic sulfide oxidation system does not suffer from this highly charged transition state, resulting in a better understanding on the effects of protonation of the peroxido with respect to the barrier of activation for oxidation. Dimethyl sulfide was used as the organic sulfide to explore a number of transition-state geometries for both anionic and neutral catalytic species. The anionic transition states located were similar to those located for halide oxidation, a nearly linear $\mathrm{S}-\mathrm{O}-\mathrm{O}$ bond angle $\left(175^{\circ}\right)$ with $\mathrm{V}-\mathrm{O}-\mathrm{O}-\mathrm{O}$ dihedral angle of $101^{\circ}$. The $\mathrm{S}-\mathrm{O}$ bond length at the transition state was $2.08 \AA$ indicating a high degree of sulfideoxygen bonding character. These geometric parameters were conserved across the four anionic transition states located, two transitions for nucleophilic attack of DMS on the left- and right-hand side of the complex with two rotational isomers of DMS per side. Protonation of the peroxido moiety of $\mathbf{1}$ yielded lower energy transitions states (0a_s, 0b_s), again exhibiting a lower degree of sulfur-oxygen bond formation leading to an earlier transition state identical to the trend observed for halide oxidation. The decrease in the barrier of activation of $8 \mathrm{kcal} / \mathrm{mol}$ is consistent with the dramatic increase in the rate of sulfide oxidation by $\mathrm{K}\left[\mathrm{VO}\left(\mathrm{O}_{2}\right)\right.$ Hheida] upon protonation. As the transition states located for the anionic catalytic species (1_s1, 1_s2, etc.) are monoanionic, there is an overestimation of the barrier of activation for this pathway, though this overestimation is less dominant that it was in the case of the dianionic halide transition states. The anionic catalytic species may still present a viable reaction path in the presence of strong hydrogen bonding or ionic effects that could stabilize the transition state. This being said, the energy differences between the 1_s and $\mathbf{0 a} \_\mathbf{s}$ or $\mathbf{0 b} \_\mathbf{s}$ are significant enough to consider the latter to be the dominant catalytic pathway.

The results presented above provide a direct correlation between the proposed transition-state geometries for VHPO's with the best known functional mimic for the same enzymes, making $\mathrm{K}\left[\mathrm{VO}\left(\mathrm{O}_{2}\right)\right.$ Hheida] a complete functional model for vanadium-dependent bromoperoxidases. As was the case for the enzyme studies, protonation of the peroxido moiety leads an early transition state and a dramatic decrease in the barrier of activation for substrate oxidation (substrate $=$ halide or sulfide). Typical features of the transition states from enzyme model system are maintained (i.e. nearly linear $\mathrm{O}-\mathrm{O}-\mathrm{S}$ angle, similar bonding distances). Additionally, we provide support to the reactivity hypothesis presented by Colpas et al. describing the halide oxidation ability of $\mathrm{K}\left[\mathrm{VO}\left(\mathrm{O}_{2}\right)\right.$ Hheida]. The early transition states generated by protonation of the complex lead to small differences in the barrier of activation for halide oxidation; in contrast to the large difference in reduction potential for chloride, bromide, and iodide.

A comparison of van der Waals reactants adducts obtained with the hydrido-peroxido species of enzyme and synthetic models show that in both cases the substrate has a suitable spatial orientation to react with the hydroperoxido moiety. In particular, in the enzyme models, the atom of the substrate which undergoes oxidation $(\mathrm{S}, \mathrm{Br}, \mathrm{I}, \mathrm{Cl})$ is weakly coordinated to the vacant coordination position of vanadium and the interaction with vanadium place the substrate in the proper spatial orientation to act as attacking group in the $\mathrm{S}_{\mathrm{N}} 2$ reaction. ${ }^{[26]}$ In the synthetic model, the role of vanadium in positioning the substrate is played by the hydroxido ligand coordinated to the metal. In fact, the structure of the pre-reaction adducts obtained with the hydroperoxido form of the synthetic complex show that the atom of the substrate that will undergo oxidation is involved in a 
hydrogen bond with the hydroxido group. Formation of this hydrogen bond positions the substrate in the proper location to act as a nucleophile in the $\mathrm{S}_{\mathrm{N}} 2$ reaction. Therefore, in both cases (enzyme and synthetic models) the vanadium complex plays a role in properly positioning the substrate in the transiently formed pre-reaction adduct.

The calculated barriers to activation for $\mathrm{K}\left[\mathrm{VO}\left(\mathrm{O}_{2}\right)^{-}\right.$ Hheida], in the case of both bromide and sulfide oxidation, are slightly higher than that calculated for the enzyme system (Table 4). The differences in the barriers of activations between the enzyme and synthetic model systems can be explained by the polarization of the peroxido bond and the torsional angle of the peroxido ligand in the transition state. The hydroperoxido moiety is more activated in the enzyme model, in particular, the $\mathrm{O}-\mathrm{O}$ bond is longer (1.467 enzyme model vs. $1.458 \AA$ synthetic model) and the peroxido bond is more polarized ( $D q=0.12$ enzyme model vs. 0.02 synthetic model). Additionally, in the transition states computed for the synthetic model, the hydroperoxido group is slightly tilted (see Figure 5 above) due to simultaneous formation of the hydrogen bond with the hydroxido group and $\mathrm{S}_{\mathrm{N}} 2$-type oxido transfer reaction. The tilting of the hydroperoxido group in the transition state is possibly another factor responsible for increasing the energy of the transition state thereby increasing the barrier to activation.

Table 4. Comparison of computed $\Delta G^{\ddagger}$ for thioether (DMS) and bromide $(\mathrm{Br})$ oxidation using the enzyme and synthetic models (energies in $\mathrm{kcal} / \mathrm{mol}$ ). All energy values are obtained as difference between TS energies and Van der Waals reactant adducts.

\begin{tabular}{lll}
\hline & Enzyme model & Synthetic model \\
\hline $\mathrm{DMS}$ & 2.9 & 9.4 \\
$\mathrm{Br}^{-}$ & 3.7 & 21.1 \\
\hline
\end{tabular}

These results demonstrate the importance of protons in catalytic process. Many vanadium catalysts have been presented in the literature for a variety of oxidations: alcohol oxidations, ${ }^{[38]}$ olefin epoxidation, ${ }^{[1,39-41]}$ sulfide oxidation,,$^{[6,7,9,11,41]}$ and phosphane oxidation..$^{[1,8]}$ Many of these processes use in-situ-derived catalytic systems from vanadium starting materials, vanadyl sulfate and vanadyl acetylacetonate being the most common, with a variety of ligands. The reported rates and turnover numbers for sulfide oxidation are significantly lower than those presented for $\mathrm{K}\left[\mathrm{VO}\left(\mathrm{O}_{2}\right)\right.$ Hheida], despite the similarities in coordination environments and terminal oxidant (hydrogen peroxide). The difference between other systems and the Hheida system is often the protonation of the peroxido ligand; which as shown herein decrease the barrier of activation and has experimentally been shown to increase the rate of reaction. To design better oxidation catalysts using vanadium, care should be taken to limit potential competitive protonation states. Additionally, steric bulk and H-bond acceptors should be added in such a way as to encourage selective protonation of one side of peroxido ligand thereby limiting the potential orientations a pro-chiral can adopt in the linear $\mathrm{S}_{\mathrm{N}} 2$ type transition state. The computational re- sults contained herein make it possible to design ligand sets capable of stereoselective oxidations using non-redox active $\mathrm{d}^{0}$ metal-peroxido coordination complexes rationally.

\section{Conclusions}

Our group has shown $\mathrm{K}\left[\mathrm{VO}\left(\mathrm{O}_{2}\right)\right.$ Hheida] to be an effective functional model and biomimetic catalyst for thioether and halide oxidation. Density functional theory was used to explore the chemically feasible pre-catalytic species, protonation states, and transition-state geometries. We have shown that coordination of the hydroxyethyl arm of Hheida is energetically favored over coordination of an exogenous donor such as acetonitrile or water. All chemically reasonable protonation states were examined on the basis of the precatalytic species. These results showed that protonation of the peroxido moiety is favored over protonation of Hheida or the oxido ligand. Transition states were located for thioether and halide oxidations (halide $=\mathrm{I}^{-}, \mathrm{Br}^{-}$, and $\mathrm{Cl}^{-}$), all transition states were $\mathrm{S}_{\mathrm{N}}{ }^{2}$-like, with a nearly linear substrate-peroxido oxygen bond angles. Protonation of the peroxido moiety dramatically reduces the barrier to activation for both thioether and halides; however barriers are insensitive to the identity of the halide. This further supports the hypothesis that the inability of $\mathrm{K}[\mathrm{VO}(\mathrm{OOH})$ Hheida] to oxidize chloride is the result of the increasing basicity of the substrate, which leads to deprotonation of the catalytically active hydroperoxido species. These results provide the necessary information regarding the transitionstate geometry and the importance of protons in the catalytic cycle to develop the class of catalysts based upon $\mathrm{K}[\mathrm{VO}(\mathrm{OOH}) \mathrm{Hheida}]$ for asymmetric oxidations.

\section{Experimental Section}

DFT geometry optimization of molecular structures were performed using the BP86 functional ${ }^{[42]}$ and an all-electron triple- $\zeta$ basis set with polarization functions on all atoms (TZVP). ${ }^{[43]} \mathrm{Cal}-$ culations were carried out using the Turbomole suite of programs $^{[44]}$ in conjunction with the resolution of identity technique. ${ }^{[45,46]}$

Optimizations of the transition-state structures were performed using a procedure based on a pseudo Newton-Raphson method. Initial starting structures of a guessed transition state were optimized by constraining the distances related to the reaction coordinate, specifically the peroxido $\mathrm{O}-\mathrm{O}$ and the substrate $\mathrm{X}-\mathrm{O}$ or $\mathrm{S}-\mathrm{O}$ (where $\mathrm{X}=\mathrm{I}^{-}, \mathrm{Br}^{-}, \mathrm{Cl}^{-} \mathrm{S}=$ sulfur of dimethyl sulfide) interatomic distances. The initial Hessian matrix was calculated at the classical level, whereas the BP86-RI/TZVP was used to compute the Hessian matrix of the minimum energy structures. Full vibrational analysis of the stationary points were carried out to ensure the presence of one eigenmode featuring an imaginary frequency corresponding to the reaction coordinate. This point was then used as a starting point for the transition-state searches, which were conducted using an eigenvector-following strategy.

Transition states are labeled on the basis of the number negative charges present on vanadium complex ( -1 or $\mathbf{0}$ ), site of protonation (Figure 6, a), and the substrate. In the case of dimethyl sulfide transition states a number has been assigned to the rotational confor- 
mation of the methyl groups, $\mathbf{P}$ indicating a methyl group parallel to the vanadium peroxido group or $\mathbf{A}$ indicating an antiparallel methyl group (Figure 6, b). A transition state corresponding to protonation of the left-side peroxido oxygen atom with dimethyl sulfide in a parallel methyl group orientation would be $\mathbf{0 a} \_\mathbf{s P}$, while the anionic transition state for bromide oxidation would be $\mathbf{1}$ _br.

a)

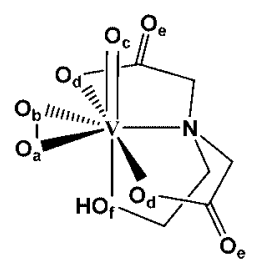

b)

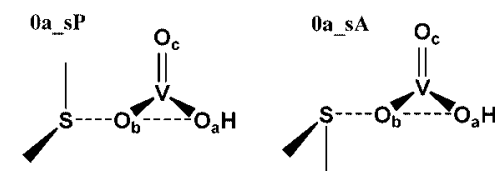

Figure 6. Illustration of the nomenclature adopted for this manuscript.

Full vibrational analysis has been carried out to characterize each stationary point. The effects of solvation were evaluated with the solvent continuous model approach $\mathrm{COSMO}^{[47,48]}$ as implemented in the Turbomole suite. The polarizable continuum medium is expected to mimic the effects of a pure solvent environment to this purpose the dielectric constant for acetonitrile $(\varepsilon=36.64)$ was used as previous studies ${ }^{[6,27]}$ have employed it as the solvent to explore the reactivity of monoperoxido oxidovanadium complexes. Corrections have been computed to estimate $\Delta G$ from $\Delta E$, by calculating roto-vibrational partition function of the different molecular species.

\section{Acknowledgments}

CJS would like thank the National Institutes of Health (NIH) (GM008597, CBI Training Program) and the Sokol International Summer Research Fellowship for funding.

[1] C. Bolm, Coord. Chem. Rev. 2003, 237, 245-256.

[2] S. A. Blum, R. G. Bergman, J. A. Ellman, J. Org. Chem. 2003, $68,153-155$

[3] G. Santoni, G. Licini, D. Rehder, Chem. Eur. J. 2003, 9, 47004708 .

[4] F. Van de Velde, I. W. C. E. Arends, R. A. Sheldon, J. Inorg. Biochem. 2000, 80, 81-89.

[5] I. Fernandez, N. Khiar, Chem. Rev. 2003, 103, 3651-3705.

[6] T. S. Smith, V. L. Pecoraro, Inorg. Chem. 2002, 41, 6754-6760.

[7] A. Barbarini, R. Maggi, M. Muratori, G. Sartori, R. Sartorio, Tetrahedron: Asymmetry 2004, 15, 2467-2473.

[8] G. Du, J. H. Espenson, Inorg. Chem. 2005, 44, 2465-2471.

[9] Y.-C. Jeong, S. Choi, Y. D. Hwang, K.-H. Ahn, Tetrahedron Lett. 2004, 45, 9249-9252.

[10] P. Plitt, H. Pritzkow, R. Kraemer, Dalton Trans. 2004, 2314 2320 .

[11] J. Sun, C. Zhu, Z. Dai, M. Yang, Y. Pan, H. Hu, J. Org. Chem. 2004, 69, 8500-8503.

[12] H. Vilter, Met. Ions Biol. Syst. 1995, 31, 325-362.

[13] A. Butler, J. N. Carter-Franklin, Nat. Prod. Rep. 2004, 21, 180 188.
[14] H. Vilter, Phytochemistry 1984, 23, 1387-1390.

[15] M. Andersson, A. Willetts, S. Allenmark, J. Org. Chem. 1997, $62,8455-8458$.

[16] R. R. Everett, J. R. Kanofsky, A. Butler, J. Biol. Chem. 1990, 265, 4908-4914.

[17] C. Rush, A. Willetts, G. Davies, Z. Dauter, H. Watson, J. Littlechild, FEBS Lett. 1995, 359, 244-246.

[18] M. Weyand, H. J. Hecht, H. Vilter, D. Schomburg, Acta Crystallogr., Sect. D. 1996, D52, 864-865.

[19] A. Messerschmidt, L. Prade, R. Wever, Biol. Chem. 1997, 378 , 309-315.

[20] A. Mueller-Fahrnow, W. Hinrichs, W. Saenger, H. Vilter, FEBS Lett. 1988, 239, 292-294.

[21] N. Pooransingh-Margolis, R. Renirie, Z. Hasan, R. Wever, A. J. Vega, T. Polenova, J. Am. Chem. Soc. 2006, 128, 5190-5208.

[22] T. Borowski, W. Szczepanik, M. Chruszcz, E. Broclawik, Int. J. Quantum Chem. 2004, 99, 864-875.

[23] J. Y. Kravitz, V. L. Pecoraro, H. A. Carlson, J. Chem. Theory Comput. 2005, 1, 1265-1274.

[24] S. Raugei, P. Carloni, J. Phys. Chem. 2005, ACS ASAP.

[25] G. Zampella, J. Y. Kravitz, C. E. Webster, P. Fantucci, M. B. Hall, H. A. Carlson, V. L. Pecoraro, L. De Gioia, Inorg. Chem. 2004, 43, 4127-4136.

[26] G. Zampella, P. Fantucci, V. L. Pecoraro, L. De Gioia, J. Am. Chem. Soc. 2005, 127, 953-960.

[27] G. J. Colpas, B. J. Hamstra, J. W. Kampf, V. L. Pecoraro, J. Am. Chem. Soc. 1996, 118, 3469-3478.

[28] P. E. M. Siegbahn, M. R. A. Blomberg, Chem. Rev. 2000, 100, 421-437.

[29] S. Niu, M. B. Hall, Chem. Rev. 2000, 100, 353-405.

[30] D. Balcells, F. Maseras, A. Lledos, J. Org. Chem. 2003, 68, 4265-4274.

[31] D. Balcells, F. Maseras, G. Ujaque, J. Am. Chem. Soc. 2005, 127, 3624-3634.

[32] A. Butler, M. J. Clague, G. E. Meister, Chem. Rev. 1994, 94 , 625-638.

[33] M. J. Clague, N. L. Keder, A. Butler, Inorg. Chem. 1993, 32, $4754-4761$.

[34] G. J. Colpas, B. J. Hamstra, J. W. Kampf, V. L. Pecoraro, J. Am. Chem. Soc. 1994, 116, 3627-3628.

[35] V. Conte, F. Di Furia, S. Moro, S. Rabbolini, J. Mol. Catal. A 1996, 113, 175-184.

[36] V. Conte, O. Bortolini, M. Carraro, S. Moro, J. Inorg. Biochem. 2000, $80,41-49$.

[37] C. Slebodnick, V. L. Pecoraro, Inorg. Chim. Acta 1998, 283, 37 43 .

[38] C. Li, P. Zheng, J. Li, H. Zhang, Y. Cui, Q. Shao, X. Ji, J. Zhang, P. Zhao, Y. Xu, Angew. Chem. Int. Ed. 2003, 42, 50635066.

[39] R. F. De la Pradilla, A. Castellanos, J. Fernandez, M. Lorenzo, P. Manzano, P. Mendez, J. Priego, A. Viso, J. Org. Chem. 2006, $71,1569-1575$.

[40] J. Hartung, S. Drees, M. Greb, P. Schmidt, I. Svoboda, H. Fuess, A. Murso, D. Stalke, Eur. J. Org. Chem. 2003, 2388 2408.

[41] A. G. J. Ligtenbarg, R. Hage, B. L. Feringa, Coord. Chem. Rev. 2003, 237, 89-101.

[42] A. D. Becke, Phys. Rev. A 1988, 38, 3098-3100.

[43] A. Schaefer, C. Huber, R. Ahlrichs, J. Chem. Phys. 1994, 100, $5829-5835$.

[44] R. Ahlrichs, M. Baer, M. Haeser, H. Horn, C. Koelmel, Chem. Phys. Lett. 1989, 162, 165-169.

[45] K. Eichkorn, F. Weigend, O. Treutler, R. Ahlrichs, Theor Chem. Acc. 1997, 97, 119-124.

[46] K. Eichkorn, O. Treutler, H. Oehm, M. Haeser, R. Ahlrichs, Chem. Phys. Lett. 1995, 240, 283-290.

[47] A. Klamt, J. Phys. Chem. 1996, 100, 3349-3353.

[48] A. Klamt, J. Phys. Chem. 1995, 99, 2224-2235.

Received: August 7, 2006 Published Online: December 15, 2006 